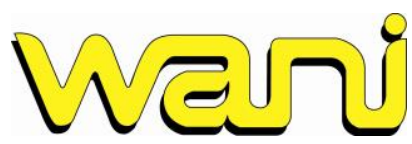

Revista del Caribe Nicaragüense

Núm. 76 | enero - junio, 2022

\title{
Epidemiología del picudo del cocotero como vector de la enfermedad del anillo rojo en plantaciones de palma africana
}

\section{Epidemiology of the coconut palm weevil as a vector of red ring disease in African palm plantations}

iD Juan Asdrúbal Flores-Pacheco ${ }^{1}$

asdrubal.flores@do.bicu.edu.ni

Elizabeth Aurora Ruiz Zolano ${ }^{2}$

asoryears.ajoh@gmail.com

Antonio José Obregón Hernández ${ }^{2}$

jobregon@cukra.com

Fecha de Recepción: 23-11-2021

Fecha de Aprobación: 04-02-2022

\section{RESUMEN}

La enfermedad del anillo rojo causada por el nematodo Bursaphelenchus (Rhadinaphelenchus) cocophilus y transmitida por el picudo americano de las palmas, Rhynchophorus palmarum, ha sido considerada la enfermedad más importante del cocotero y la palma aceitera en América tropical, y todavía hoy es común en algunas plantaciones. El progreso de los síntomas puede ser muy rápido (forma aguda) y la palma afectada puede morir en unos pocos meses después de aparecer los primeros síntomas (amarillamiento progresivo y secamiento de las hojas empezando por las más viejas). En el otro extremo de un continuo de síntomas, las hojas más jóvenes emergen cortas y con varios tipos de malformaciones, pero las plantas pueden no morir en varios años (forma crónica). La presente investigación tiene como objetivo central analizar la dinámica poblacional del picudo de cocotero (Rhynchophorus palmarum) como vector de la enfermedad del anillo rojo; esta afección es causada por el nematodo Bursaphelenchus cocophilus, el cual se localiza en plantaciones de palma africana (Elaeis guianensis) localizadas en el municipio de Kukra Hill. Empleando dos tipos de trampas para la colecta de adultos de R. palmarum, y la cirugía para la extracción de su fase larvaria (esto combinado con el embudo de Baerman y el mapa de niveles críticos de la incidencia de este coleóptero), permitieron realizar el análisis epidemiológico en torno a la presencia de la enfermedad del anillo rojo ocasionada por $R$. cocophilus. No se encuentran pruebas estadísticas ni materiales (aislamiento del nematodo o presencia de plantas enfermas) que indiquen su presencia en las plantaciones de palma africana en Kukra Hill. Deben

${ }^{1}$ Bluefields Indian \& Caribbean University, Director de Investigación y Posgrado, Bluefields, Nicaragua.

${ }^{2}$ Bluefields Indian \& Caribbean University, Licenciada en Ecología de los Recursos Naturales, Bluefields, Nicaragua. 


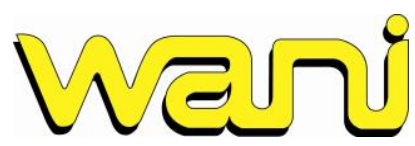

Revista del Caribe Nicaragüense

Núm. 76 | enero - junio, 2022
e-ISSN 2308-7862

Bluefields Indian \& Caribbean University (BICU)

DOI: https://doi.org/10.5377/wani.v38i76.13377

http://revistas.bicu.edu.ni/index.php/wani

wani@bicu.edu.ni

realizarse más estudios, durante más tiempo y con mayor número de repeticiones, para la corroboración y/o cambio en los hallazgos de este trabajo.

Palabras claves: Nematología, control de plagas, vector, fitopatología, Caribe de Nicaragua

\section{ABSTRACT}

The disease of the red ring, caused by the nematode Bursaphelenchus (Rhadinaphelenchus) cocophilus and transmitted by the American palm weevil, Rhynchophorus palmarum, has been considered the most important disease of the coconut palm and oil palm in tropical America, and it is still common today in some plantations. The progress of symptoms can be very rapid (acute form), and the affected palm can die within a few months after the first symptoms appear (progressive yellowing and drying of the leaves starting with the oldest). At the other end of a continuum of symptoms, the younger leaves emerge short and with various types of malformations, but the plants may not die in several years (chronic form). The main aims of this research are to analyze the population dynamics of the coconut palm weevil (Rhynchophorus palmarum) as a vector of the red ring disease caused by the nematode (Bursaphelenchus cocophilus) in plantations of African Palm Oils (Elaeis guianensis), located in the municipality of Kukra Hill. Using two types of traps for the collection of adults of R. palmarum and surgery for the extraction of its larval phase, this combined with the Baerman funnel and the map of critical levels of the incidence of this coleopter allowed to perform the epidemiological analysis of the presence of Red Ring disease caused by $R$. cocophilus. There are no statistical or material tests (isolation of the nematode or presence of diseased plants) that indicate its presence in the African palm plantations in Kukra Hill. More studies should be done longer and with more repetitions for the corroboration and / or change of the findings of this work.

Keywords: nematology, pest control, vector, phytopathology, Caribbean of Nicaragua.

\section{AISANKA PRAHNI RA}

Matka dinka pauni siknis ka yula kum mita baikisa, Bursaphelenchus (Rhadinaphelenchus) cocophilus baku sim baha siknis ka daiwan lupia kum plis kum wina plis wala ra brihuisa naha nina ba Rhynchophorus palmarum, naha siknis ka ba sakan sa kau saura kuku dusa ra baku sim lat uhum dusa ra America tropical ra. Naiwa sin siknis ka ba ailal iwisa. Kuku an uhum ra alkan tem istikira sa, baku sin siknis ka dusa almuk ra alkan tem dusa ba iskiria pruisa, maka dusa wahia lalahni dinkan tem pat lawisa, dusa wahma ra wahia raya pawisa tem sirpi takisa baku sim wahia ba aihka takisa, dusa wahma ra siknis ka wal istikira pruras sa. Naha Laki kaikan ba bapan ka brisa laki kaikaia matka dinka pauni siknis ka, naha laki kaikan ba Africa Uhum dusa nasla ra Kukra Hill Tawan kara siknis ka iwisa sapa. Yawan yula ba alkaikan sat wal nanih daukan baku sin biara baikaia dukiara baha ba ai biara ra día brisa laki kaikaia wisi, baku sin yawan nani kaikan nahki mita naha yula ba iwisa. Nata ra Kukra Hill tawankara uhum dusa nani laki kaikan tem yula nani uba kaikras kan, baha mihta nitsa taim wala ra kli bal laki kaikaia yula ba iwisa apia kaka apu naha tawankara. 


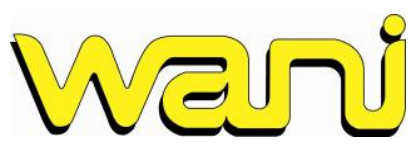

Revista del Caribe Nicaragüense

Núm. 76 | enero - junio, 2022
e-ISSN 2308-7862

Bluefields Indian \& Caribbean University (BICU)

DOI: https://doi.org/10.5377/wani.v38i76.13377

http://revistas.bicu.edu.ni/index.php/wani

wani@bicu.edu.ni

Bila bak sakan nani: Yula laki kaikaia, yula wapni bankaia, Dusa lakan ka, Nicaragua Caribe Baiki sakan

Para citar este artículo (APA): Flores-Pacheco J.A.; Ruiz Zolano, E. \& Obregón Hernández A.J. (2022). Epidemiología del picudo del cocotero como vector de la enfermedad del anillo rojo en plantaciones de palma africana. Wani (76), 70-83. https://doi.org/10.5377/wani.v38i76.13377

\section{INTRODUCCIÓN}

Un cultivo perenne como la palma africana, con el transcurso del tiempo se va convirtiendo de muy simple a más complejo. En las plantaciones ubicadas en el municipio de Kukra Hill, según la forma como se contribuya al manejo, menores serán los incrementos de las poblaciones de plagas (BICU, 2011). La palma de aceite, al igual que la mayoría de los cultivos agrícolas, sufren de problemas fitosanitarios -plagas y enfermedades- tales como los picudos (Rhynchophorus palmarum). Éstos se presentan como plagas de importancia económica dado que el incremento de sus poblaciones se produce en un largo periodo, y las grandes pérdidas de flecha seca pueden alcanzar el $90 \%$ en las áreas afectadas. Toda la planta completa presenta amarillamiento (Lastres \& Soza, 2009).

Para evitar la propagación inmediata de la población de picudo, ésta puede ser regulada mediante la captura del adulto por medio de trampas colocadas con un atrayente (Chinchilla, 2003). La fertilización es uno de los elementos primordiales que no fallan en el manejo; al igual que las trampas, están hechas de envases de 12 litros a los cuales se les colocan pedazos de sacos para evitar que el picudo logre salirse del envase; también se les agrega un litro de melaza acompañado con feromonas que es un atrayente para el picudo.

El trampeo se realiza cada ocho días para contabilizar la cantidad de picudo por trampa. Gracias a los métodos utilizados se han logrado buenos resultados (REPcar - BICU, 2014). El rango máximo de picudo es de 2.5 picudos por trampa promedio semanal. Cuando el estado es crítico, las revisiones se acortan a ocho días. Así mismo se incrementa el número de trampas por lotes, para evitar el incremento de esta plaga causante de la flecha seca (BICU, 2011).

Cepeda (1996) menciona que los nematodos, al igual que los virus, son considerados los enemigos ocultos de los agricultores de palma africana, sin embargo, los síntomas ocasionados pueden ser similares a los generados por hongos y bacterias fitopatógenas, los cuales no permiten dar un diagnóstico exacto en campo. Es por esto que día a día diversos investigadores tratan de dar respuestas usando métodos sencillos, procurando ahorro de tiempo, extracción eficiente, fácil, y manipulación segura de los nematodos. Los adultos de $R$. palmarum presentan una gran variación en tamaño de 20 a 41 milímetros los sexos pueden ser normalmente diferenciados, considerando que el macho tiene un penacho de setas sobre el rostum. El ciclo de vida de huevo a adulto ocurre entre 80 y 160 días, y el adulto puede vivir por tres meses o más. La hembra pone entre 10 y 48 huevos cada día durante un periodo de ocho a once días y algunas pueden poner hasta 60 huevos durante los primeros tres días (Oehlschlager et al., 2006).

Las hembras adultas del picudo son atraídas por compuestos volátiles que emanan de palma con heridas o pudriciones en las cuales ovopositan. Las plantas en las cuales ya ha avanzado 


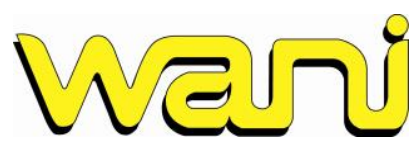

Revista del Caribe Nicaragüense

Núm. 76 | enero - junio, 2022
e-ISSN 2308-7862

Bluefields Indian \& Caribbean University (BICU)

DOI: https://doi.org/10.5377/wani.v38i76.13377

http://revistas.bicu.edu.ni/index.php/wani

wani@bicu.edu.ni

drásticamente la enfermedad anillo rojo han sido erradicadas para evitar los albergues del picudo, puesto que, si esto no se hace, el insecto y la enfermedad se propagan de manera rápida. Si esta enfermedad avanza, nos veremos expuestos al desempleo, pues esta actividad es la única fuente de trabajo que existe en el municipio de Kukra Hill (Sommer et al., 1994).

La palma africana es la de mayor rendimiento en producción de aceite por hectáreas; por esta razón, la generación de información e investigación sobre su estructuración y organización es muy importante para dar a conocer cómo aumentar los rasgos de adaptabilidad de esta especie, y así ser competitivos en el mercado nacional e internacional (Taussig, 2018). La incidencia de la enfermedad puede ser mantenida a un bajo nivel si se sigue una estrategia de manejo integrado, la cual incluye: eliminación temprana de palmas enfermas, destrucción de sitios de reproducción del vector y reducción de población adulta (Oehlschlager et al., 2006).

Esta investigación analizó la dinámica poblacional del picudo del cocotero (Rhynchophorus palmarum) como vector de la enfermedad del anillo rojo causada por el nematodo Bursaphelenchus cocophilus en plantaciones de palma africana (Elaeis guianensis) localizadas en el municipio de Kukra Hill.

\section{MATERIALES Y MÉTODOS}

\section{Localización}

El área de investigación está ubicada en la comunidad Flor de Pino del municipio Kukra Hill, en las plantaciones de palma africana de la Cukra Development Corporation S.A., conocida como palma aceitera. El estudio se realizó durante el periodo de diciembre de 2018 a enero de 2019. La zona de estudio está ubicada en el trópico húmedo, por lo cual es común tener temperaturas de $24^{\circ}$ $\mathrm{C}$ a $30^{\circ} \mathrm{C}$, con precipitaciones de 2,000 $\mathrm{mm}$ a 4,000 $\mathrm{mm}$ anuales. La zona es baja y pantanosa, característica de la región, con una altura que no excede los $30 \mathrm{msnm}$ (metros sobre el nivel del mar), con suelos entisoles e insectisoles: el pH promedio es de seis; los vientos son ligeramente moderados con dirección de Este a Oeste.

De acuerdo con los objetivos planteados, esta investigación es de tipo exploratorio, de carácter descriptivo debido a que el estudio se basa en la epidemiología del picudo (Rhynchophorus palmarum) como vector del anillo rojo, una enfermedad causada por el nematodo Bursaphelenchus cocophilus en plantaciones de palma africana (Elaeis guianensis) localizadas en el municipio de Kukra Hill, Región Autónoma de la Costa Caribe Sur (RACCS) de Nicaragua. Estas plantaciones presentan datos de daños causales. La investigación tiene como objetivo dar a conocer la epidemiologia del vector del anillo rojo ocasionada por el nematodo Bursaphelenchus cocophilus. El intento de solventar este problema determina la naturaleza de nuestra investigación como aplicada. El marco de la exploración estuvo enfocado en plantas con presencia de flecha seca, ya que las plantas enfermas le sirven al picudo como huésped mientras la enfermedad avanza de manera drástica. La falta de intervención de cirugía para contrarrestar la propagación en las 
Wer

Revista del Caribe Nicaragüense

Núm. 76 | enero - junio, 2022
e-ISSN 2308-7862

Bluefields Indian \& Caribbean University (BICU)

DOI: https://doi.org/10.5377/wani.v38i76.13377 http://revistas.bicu.edu.ni/index.php/wani

plantaciones de palma africana en condiciones ambientales, la clasifica también como una investigación de campo.

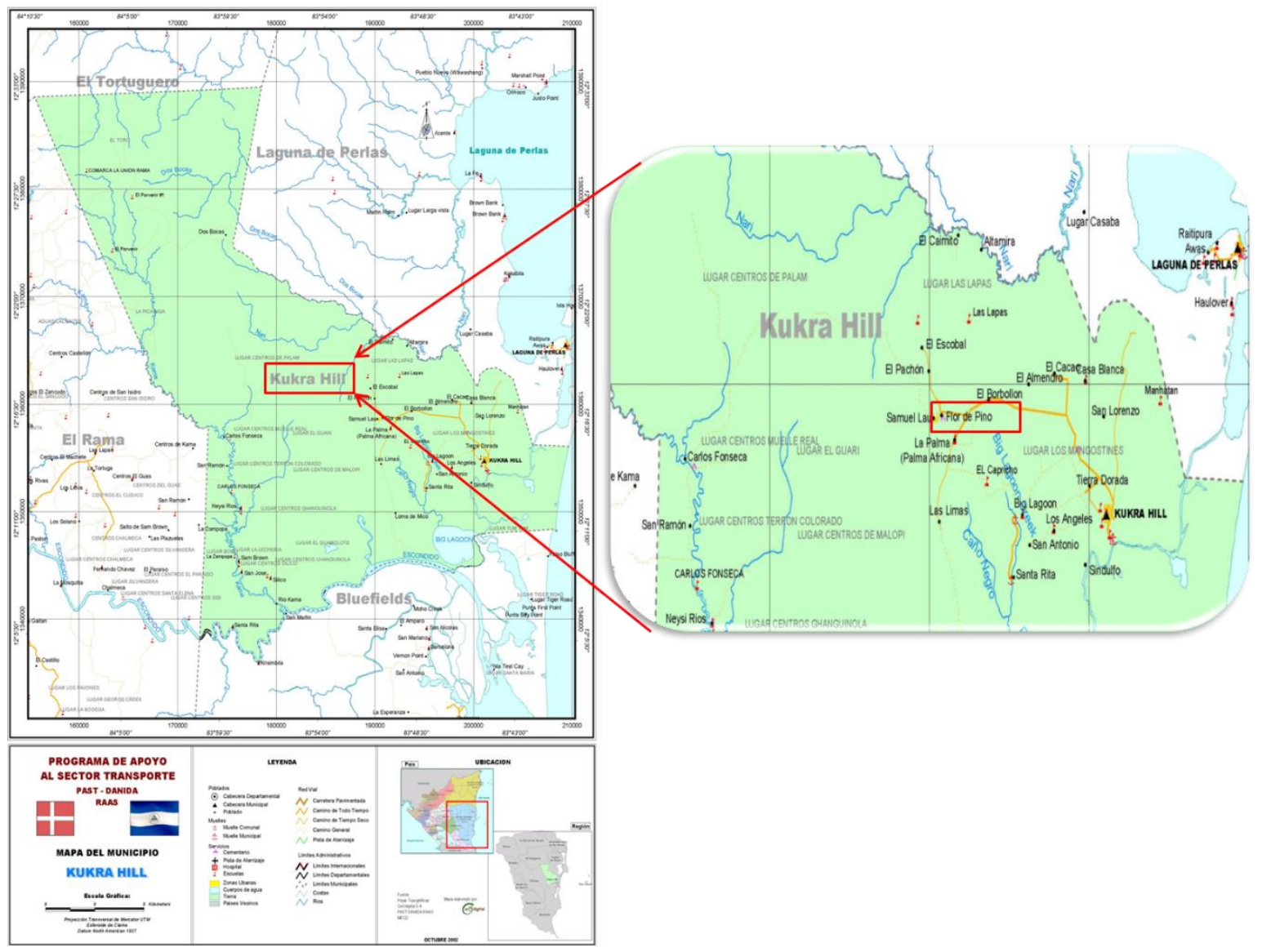

Figura 1. Ubicación del estudio

\section{Metodología de muestra}

Se seleccionó la parcela denominada lote 50, porque se sospechó la presencia de la enfermedad del anillo rojo en las plantas ahí establecidas. La parcela se dividió en cinco subparcelas de una hectárea cada una, para una intensidad de muestra del $10 \%$ del total de 50 ha correspondientes al lote 50 ubicado en la comunidad Flor de Pino y perteneciente a la Cukra Development Corporation (CDC). En cada subparcela fueron muestreadas las trampas colocadas por la CDC para el control del picudo: 1) Melaza + Cipermetrina y 2) Melaza.

En cada trampa se registró su tipo y el contenido de picudos, detallando las variables de sexo, longitud $(\mathrm{mm})$ y diámetro $(\mathrm{mm})$ del cuerpo. Aprovechando la actividad denominada cirugía fueron colectadas 50 larvas a las cuales estas variables les fueron tomadas de forma inmediata a su 
Wer

Revista del Caribe Nicaragüense

Núm. 76 | enero - junio, 2022
e-ISSN 2308-7862

Bluefields Indian \& Caribbean University (BICU)

DOI: https://doi.org/10.5377/wani.v38i76.13377

http://revistas.bicu.edu.ni/index.php/wani

wani@bicu.edu.ni

extracción del tronco de la planta intervenida. También se registró el promedio diario de precipitación en la parcela del estudio.

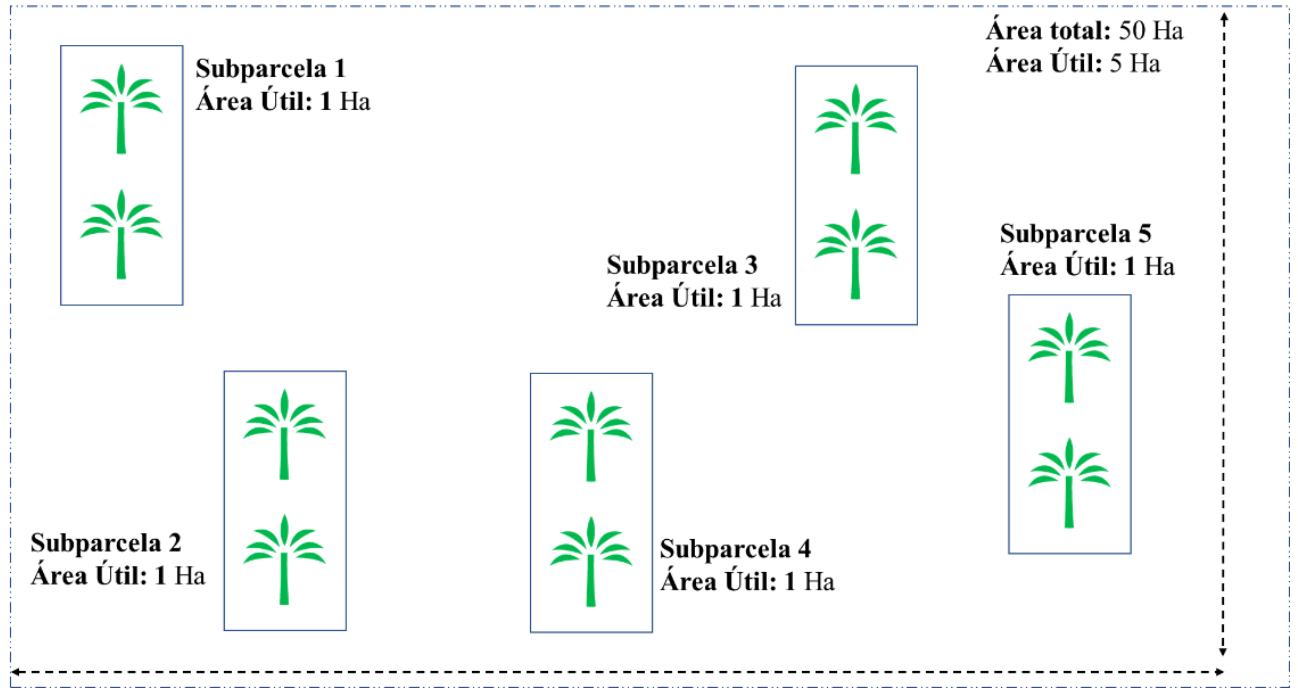

Figura 2. Posición de las parcelas de muestreo en el lote 50 de la Cukra Development Corporation S.A. en Kukra Hill.
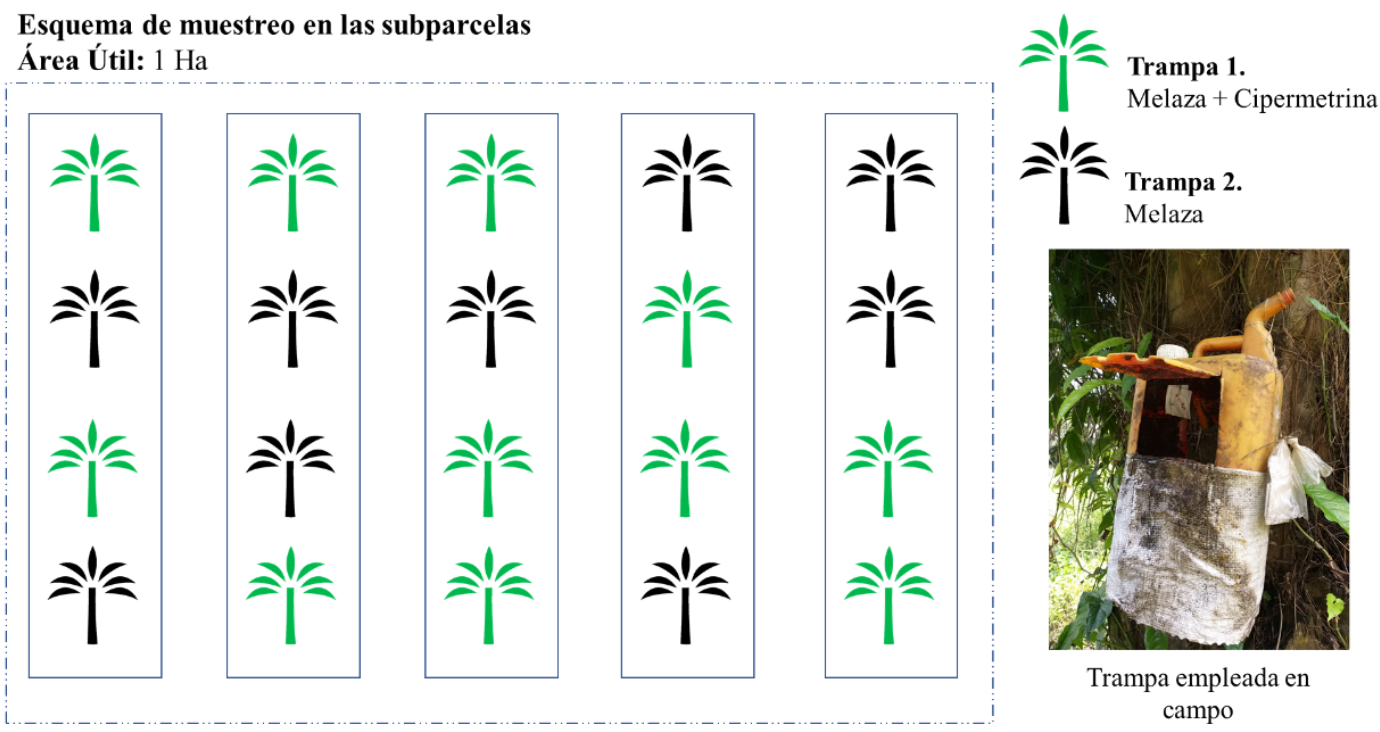

Figura 3. Esquema de la distribución de las trampas y tipos de trampas en cada subparcela de muestreo.

\section{Extracción de nematodos}

Se empleó la técnica del Embudo de Baermann, que consiste en la colocación de una hoja de papel toalla en el tamiz sobre un recipiente (Erlenmeyer). El papel toalla se humedece hasta que se 


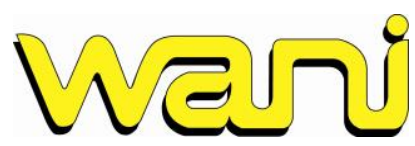

Revista del Caribe Nicaragüense

Núm. 76 | enero - junio, 2022
e-ISSN 2308-7862

Bluefields Indian \& Caribbean University (BICU)

DOI: https://doi.org/10.5377/wani.v38i76.13377 http://revistas.bicu.edu.ni/index.php/wani wani@bicu.edu.ni

adhiere al recipiente. En tal momento se procedió a la extracción del tracto digestivo de las larvas y de los picudos adultos, diferenciándolos por sexo. Esta actividad se realizó en el laboratorio de Biología del Centro de Investigaciones Acuáticas de BICU en la ciudad de Bluefields. Las larvas y picudos adultos fueron colectados el mismo día de la evisceración, conservándose en hielo desde el momento de su colecta hasta el momento de la extracción de sus entrañas. Para esta labor se emplearon pinzas entomológicas y bisturí 24 de uso médico. Se tomó cada individuo con una pinza para luego realizar un corte longitudinal en el abdomen, para la extracción del tracto digestivo. El mismo se colocó en el papel toalla humedecido.

Este material se volvió a humedecer hasta cubrirlo completamente con agua destilada dejada en reposo por un periodo de veinticuatro horas. Transcurrido este tiempo se decantó el contenido del embudo en un Erlenmeyer limpio. El líquido fue agitado para extraer una muestra de $10 \mathrm{ml}$, con el objetivo de analizarla en el hemocitómetro (cámara Thoma) con miras al conteo de los nematodos encontrados.

Para la identificación de los nematodos se siguieron las siguientes claves taxonómicas: forma y tamaño del estilete, forma y tamaño del bulbo basal, órganos reproductivos, disposición, forma y tamaño de la cola, esófago y si hay o no trasladación y movimientos del nematodo.
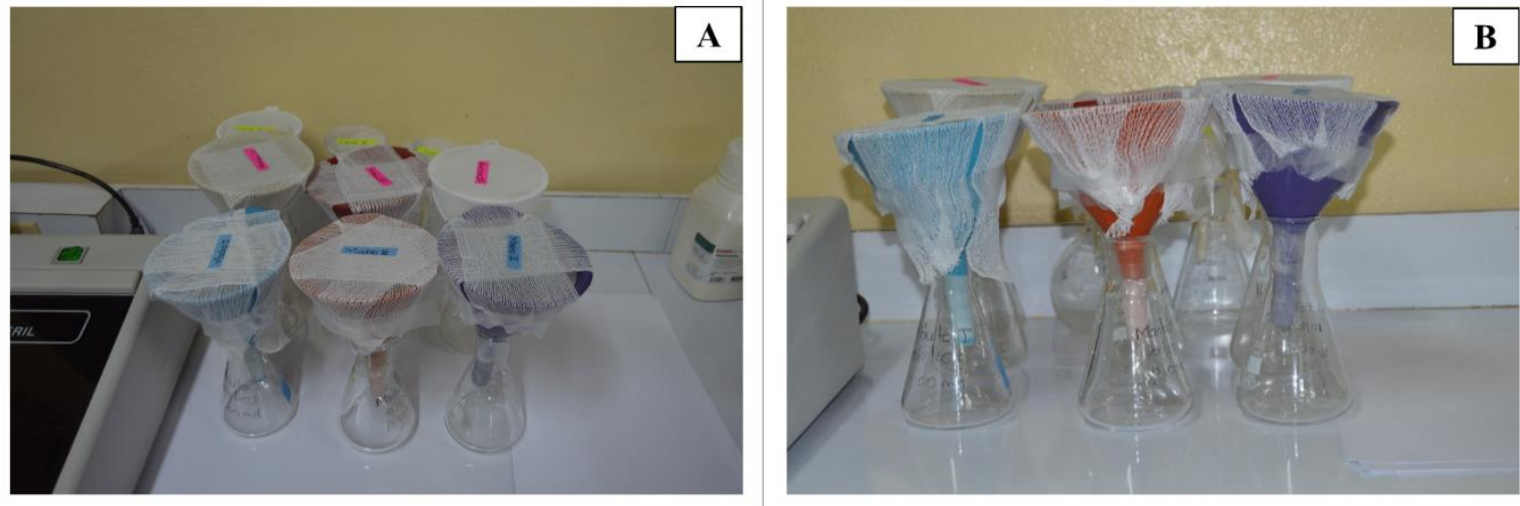

Figura 4. Embudo de Baermann; A) Clasificación en base a sexo; B) Vista general

Tabla 1. Operacionalización de las variables

\begin{tabular}{cccc}
\hline Variables & Instrumento de medición & Unidades & $\begin{array}{c}\text { Frecuencia de } \\
\text { monitoreo }\end{array}$ \\
\hline
\end{tabular}

Precipitación

Sexo del picudo
Pluviómetro

Claves taxonómicas por inspección visual
Milímetros diarios (mm/diario)

Hembra

Macho
Diario

Única 
Wer

Revista del Caribe Nicaragüense

Núm. 76 | enero - junio, 2022

DOI: https://doi.org/10.5377/wani.v38i76.13377 http://revistas.bicu.edu.ni/index.php/wani wani@bicu.edu.ni

\begin{tabular}{llll}
\hline \multicolumn{1}{c}{ Variables } & Instrumento de medición & Unidades & $\begin{array}{c}\text { Frecuencia de } \\
\text { monitoreo }\end{array}$ \\
\hline $\begin{array}{l}\text { Densidad poblacional } \\
\text { (nivel crítico) }\end{array}$ & $\begin{array}{l}\text { Relación de individuos/ } \\
\text { capturas por unidad de } \\
\text { área y/o planta }\end{array}$ & Adimensional & Semanal \\
$\begin{array}{l}\text { Presencia / Ausencia de } \\
\text { nematodo }\end{array}$ & $\begin{array}{l}\text { Método del embudo de } \\
\text { Baermann }\end{array}$ & Adimensional & Única \\
\hline
\end{tabular}

\section{Análisis estadístico}

Los datos obtenidos de las diferentes mediciones descritas en el capítulo anterior serán ingresados a una base de datos digital en el programa estadísticos SPSS 26, para su respectiva interpretación estadísticas (prueba de homogeneidad de medias, ANOVA y correlaciones) y su representación gráfica.

\section{RESULTADOS Y DISCUSIÓN}

Relación de las condiciones ambientales (precipitación) con la dinámica poblacional de $R$. palmarum

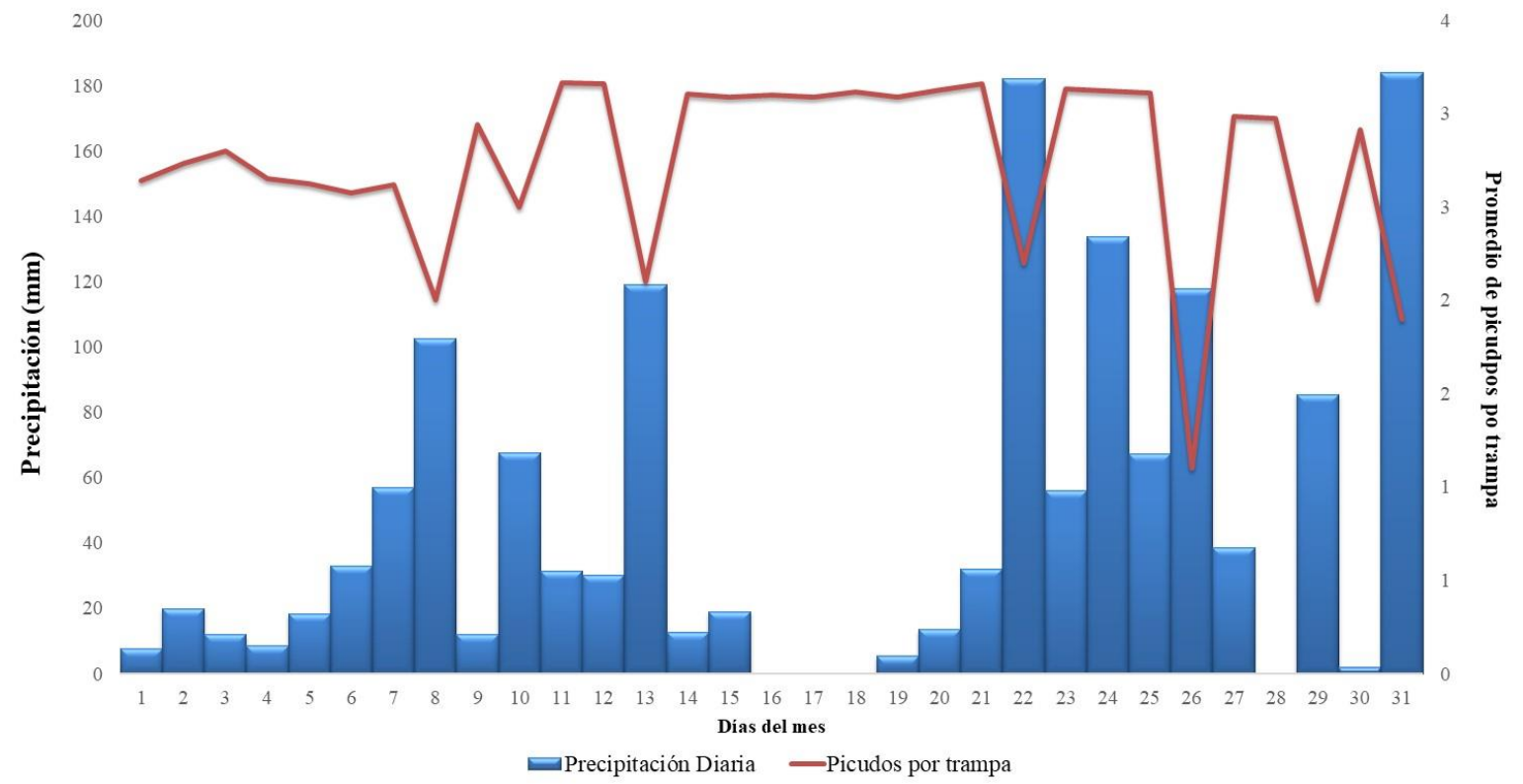

Figura 5. Relación de la precipitación $(\mathrm{mm})$ con la cantidad de individuos $R$. palmarum capturados en plantaciones de palma africana en Kukra Hill. 


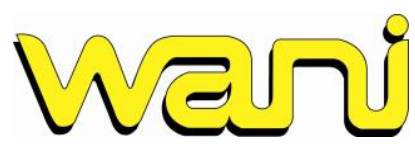

Revista del Caribe Nicaragüense

Núm. 76 | enero - junio, 2022

En la publicación de Cave, Trabanino \& Pitty (2013) se expresa que las lluvias en las zonas del Caribe de la región centroamericana representan uno de los factores naturales de mayor importancia en el control de poblaciones insectiles (huevecillos, larvas y adultos). Esto por acción mecánica directa, o por ahogamiento en el caso de acumulación de aguas. Sin embargo, la disminución e irregularidad de las lluvias por efecto del cambio climático ha venido a favorecer el establecimiento de plagas primarias u oportunistas en agroecosistemas donde no existían o únicamente eran una plaga secundaria (Diez Casero, 2013), tal cual está pasando con el picudo en las plantaciones de palma africana de Kukra Hill.

El análisis de los datos demostró la existencia de una correlación inversamente proporcional (0.79) entre el número de picudos colectados en las trampas cuando las precipitaciones son más intentas y de larga duración. Este control poblacional es de gran impacto, pues llega a regular hasta el 50\% del total de individuos/capturas, lo que se traduce en una amplia y significativa reducción de posturas de huevecillos que se convertirían en larvas las cuales acabarían con las plantaciones de palma (Taussig, 2018). No se debe obviar que tanto las larvas como los adultos de R. palmarum son vectores de $B$. cocophilus el cual a su vez es capaz de alojarse en el tracto digestivo de individuos libres, para ser transportado a plantaciones sanas (Oehlschlager et al., 2006).

Nivel crítico de las poblaciones del picudo del cocotero (Rhynchophorus palmarum) en plantaciones de palma africana (Elaeis guianensis)

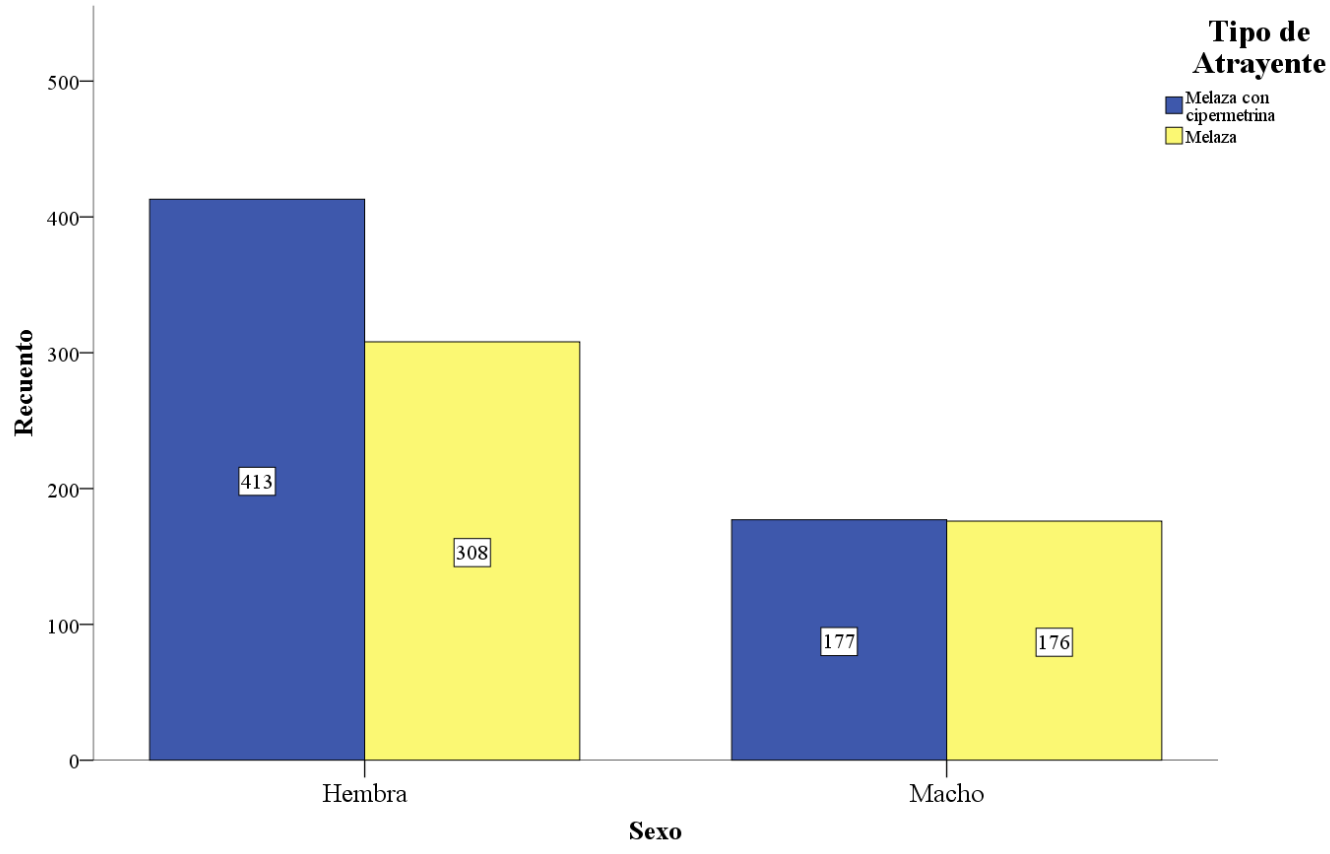

Figura 6. Capturas de adultos de $R$. palmarum en función del atrayente utilizado.

En la fase de captura de los individuos de $R$. palmarum, las trampas con la combinación de melaza + cipermetrina tuvieron un índice mayor de captura $(\mathrm{F}=6.170 ; \mathrm{gl}=8 ; \mathrm{P}<0.000)$ en relación con la trampa que únicamente utilizó melaza. Este factor puede deberse a que el añadir cipermetrina a la 


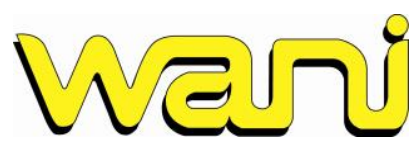

Revista del Caribe Nicaragüense

Núm. 76 | enero - junio, 2022
e-ISSN 2308-7862

Bluefields Indian \& Caribbean University (BICU)

DOI: https://doi.org/10.5377/wani.v38i76.13377

http://revistas.bicu.edu.ni/index.php/wani

wani@bicu.edu.ni

melaza intensifica su olor y disminuye su viscosidad, lo cual la hace más atractiva (Serra et al., 2005).

Es interesante destacar que en esta misma trampa también cae mayor cantidad de hembras de esta especie, lo cual indica que el olor del producto fermentado es un factor determinante de su éxito. Esto coincide con investigaciones realizadas por investigadores de BICU en el manejo de dípteros (Flores-Pacheco et al., 2019).

Tabla 2. Variables morfométricas de los estados de desarrollo de $R$. palmarum posibles vectores de $B$. cocophilus.

\begin{tabular}{cccc}
\hline Estado & Sexo & Longitud $(\mathbf{m m})$ & Diámetro $(\mathbf{m m})$ \\
\hline Larva & ------ & 41.34 & 21.70 \\
& Macho & 43.50 & 15.76 \\
Adulto & Hembra & 44.92 & 14.96 \\
\hline
\end{tabular}

La presencia de parásitos como los nematodos implica posibles afectaciones en el desarrollo de las larvas de $R$. palmarum, lo cual se traduce en adultos de tamaño ampliamente variantes. Se cree que esto es una estrategia que el organismo utiliza para que su vector se alimente de más plantas, en busca de aumentar su diseminación (Luc et al., 2005). Esta posibilidad se descarta en la población de $R$. palmarum población en las plantaciones de palma africana en Kukra Hill, ya que no se presentan estas variaciones significanticas $(\mathrm{F}=0.414 ; \mathrm{gl}=8 ; \mathrm{P}<0.912)$. Por el contrario fueron caracterizadas poblaciones estables y homogéneas que deben alertar a la empresa Cukra Development Corporation S.A., pues, la principal plaga insectil de su cultivo industrial se ha establecido con tal intensidad que las medidas de manejo convencionales y aisladas tendrán resultados limitados en la erradicación de las poblaciones de adultos y larvas (Been \& Schomaker, 2000).

Tómese en cuenta que las prácticas inadecuadas de uso sin control de insecticidas en las comunidades productivas de Kukra Hill son un potencial factor para el establecimiento de poblaciones insectiles con mayores niveles de tolerancia a los pesticidas, incrementando de esta forma los costos de manejo y los riesgos laborales y ambientales (Flores-Pacheco, Mairena, \& Espluga, 2013). En todo caso, la estrategia de control debe dirigirse a la reducción de poblaciones de adultos de $R$. palmarum, basado en que éstos poseen mayor movilidad y rango de dispersión, y sus larvas son de difícil manejo al estar en el interior de la palma, antes de emerger como adultos maduros (Landero-Torres et al., 2015).

Al contrastar el mapa de los niveles críticos de la incidencia de $R$. palmarum en las plantaciones de E. guineensis en Kukra Hill debe esperarse que, a mayor nivel crítico de las poblaciones de este coleóptero, aumenten directamente las proporciones de B. cocophilus; sin embargo, esto no sucede $\left(\mathrm{R}^{2}=-0.87\right)$. Este comportamiento es explicado únicamente por la ausencia del nematodo en la 
Wer

Revista del Caribe Nicaragüense

Núm. 76 | enero - junio, 2022
e-ISSN 2308-7862

Bluefields Indian \& Caribbean University (BICU)

DOI: https://doi.org/10.5377/wani.v38i76.13377 http://revistas.bicu.edu.ni/index.php/wani wani@bicu.edu.ni

plantaciones y, por ende, la imposibilidad que $R$. palmarum sea un vector activo de éste (Franco, 2004).

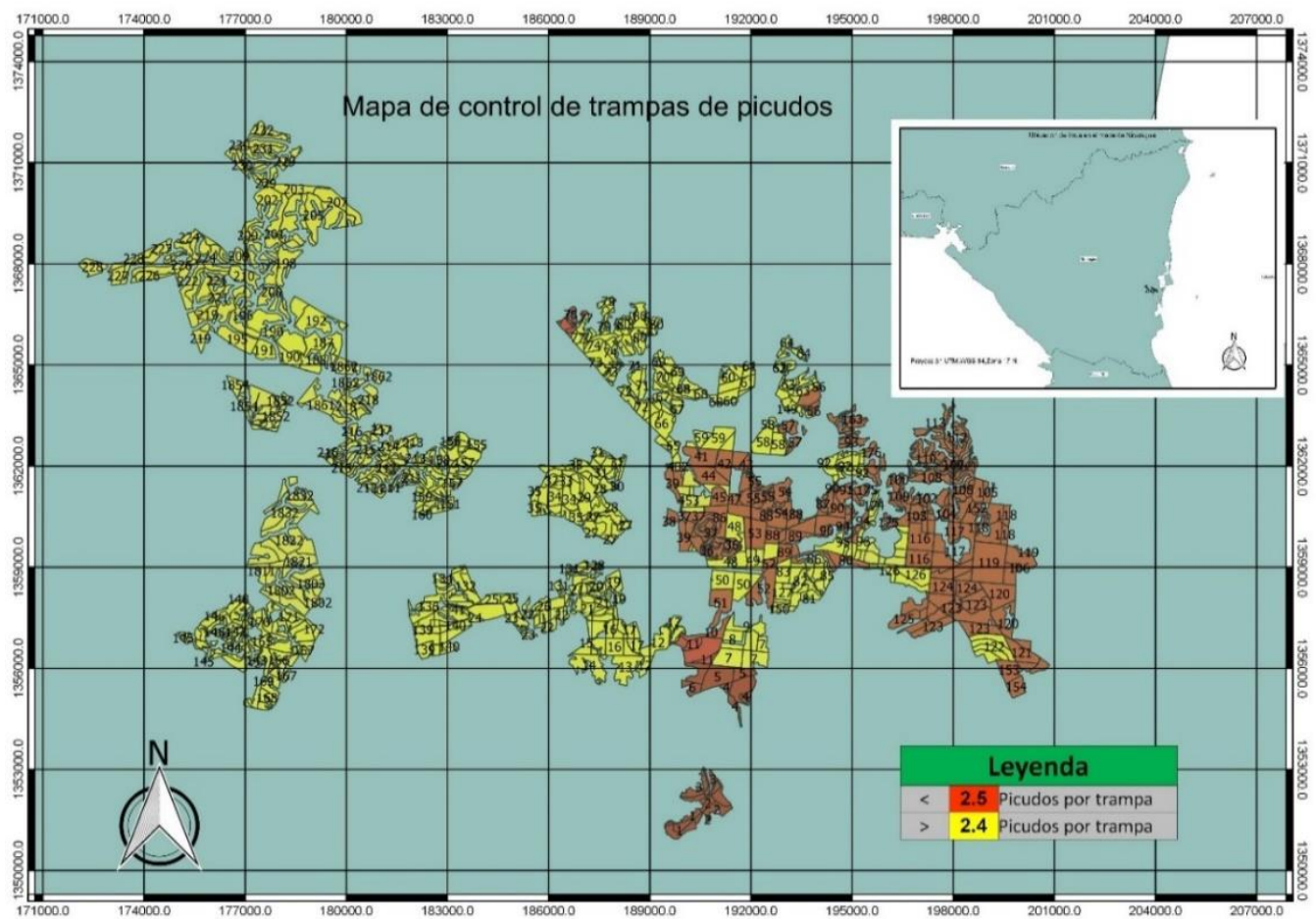

Figura 7. Nivel crítico de $R$. palmarum en función de su densidad poblacional en plantaciones de $E$. guineensis en Kukra Hill.

Estos datos son corroborados por los resultados de la extracción de parásitos internos y externos con el método del embudo de Baerman (Cadenas, 2008) expresados en la tabla 4. Con esto toma fuerza la posibilidad que las aparentes incidencias de anillo rojo en estas plantaciones sea realmente una deficiencia nutricional asociada a la carencia de potasio y/o sodio (Benavides et al., 2002).

Presencia/ausencia del nematodo (Bursaphelenchus cocophilus) en insectos (larvas y adultos) colectados en las plantaciones de palma africana (Elaeis guianensis) en Kukra Hill

Tabla 3. Determinación de la presencia de parásitos en las poblaciones de $R$. palmarum colectados en plantaciones de E. guineensis en Kukra Hill

\begin{tabular}{lccccccc}
\hline \multirow{2}{*}{ Estadio } & Sexo & & Parásitos externos & \multicolumn{2}{c}{ Parásitos internos } & \multicolumn{2}{c}{$\begin{array}{c}\text { Bursaphelenchus } \\
\text { cocophilus }\end{array}$} \\
\cline { 5 - 8 } & & Si & No & Si & No & Si & No \\
\cline { 4 - 8 } Larva & 0 & 0 & 0 & 0 & 0 & 0 \\
\multirow{2}{*}{ Adulto } & Macho & 22 & 28 & 31 & 19 & 0 & 0 \\
& Hembra & 18 & 32 & 24 & 26 & 0 & 0 \\
\hline
\end{tabular}




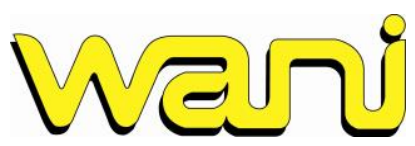

Revista del Caribe Nicaragüense

Núm. 76 | enero - junio, 2022
e-ISSN 2308-7862

Bluefields Indian \& Caribbean University (BICU)

DOI: https://doi.org/10.5377/wani.v38i76.13377

http://revistas.bicu.edu.ni/index.php/wani wani@bicu.edu.ni

Es conocido que el nematodo B. cocophilus debe ingresar al tracto digestivo de R. palmarum para poder ser diseminado por éste (Perry \& Moens, 2013) por lo cual se buscó la presencia de este parásito en el tracto digestivo tanto de larvas como de adultos; estos últimos se dividieron entre hembras y machos. En ambos casos se empleó el método del embudo de Baerman (Echandi, 1967). Las larvas no presentaron parásitos internos (tabla 4) (observación con el estereoscopio) ni la presencia de nematodos en el agua decantada analizada en el hemocitómetro veinticuatro horas posterior a la aplicación del método del embudo de Baerman (Leslie et al., 2006). En el caso de los adultos hembras y machos, éstos presentaron parásitos externos del tipo de ácaros rojos (Raoiella indica) (Mendonça et al., 2007), siendo ésta una plaga secundaria de importancia reducida en el cultivo de palma africana (BICU, 2011). Los parásitos internos, a pesar de no haber podido ser identificados, sus características no corresponden a las de ningún nematodo fitófago o de interés agrícola reportado; entre ellos, B. cocophilus, por lo cual se descarta su presencia (López, 1980).

Estos datos permiten tener mayor certeza en cuanto a la ausencia de $B$. cocophilus en las plantaciones de E. guineensis en Kukra Hill; también determinar que R. palmarum no es un vector activo del causante de la enfermedad del anillo rojo, lo cual reduce drásticamente su posibilidad de presencia en estas plantaciones. Esto fortalece la hipótesis sobre el manejo y régimen de fertilización en relación con las condiciones edafoclimáticas locales, para el control de la enfermedad del anillo rojo.

\section{CONCLUSIONES}

Redactar las conclusiones derivadas de los resultados relevantes de la investigación relacionados con los objetivos e hipótesis planteados. Se debe indicar de manera categórica, breve y precisa las aportaciones concretas al conocimiento apoyadas por los resultados demostrables y comprobables del propio trabajo. Para su redacción evite enumerar las conclusiones ni emplear abreviaturas a fin de que el lector pueda entender con claridad su contenido.

\section{CONFLICTO DE INTERESES}

Los autores declaran no tener conflicto de intereses.

\section{REFERENCIAS}

Been, T. H., \& Schomaker, C. H. (2000). Development and evaluation of sampling methods for fields with infestation foci of potato cyst nematodes (Globodera rostochiensis and G-pallida). Phytopathology, 90(6), 647-656. https://doi.org/10.1094/PHYTO.2000.90.6.647

Benavides, A., Ramirez, H., Robledo, V., Maiti, R., Cronejo, E., Hernández, J., A., S., Mendoza, R., Samaniego, E., Ramírez, J., Bacopulus, E., Aguilera, A., \& Fuentes, L. (2002). Ecofisiología y Bioquímica del Estrés en Plantas (A. B. Mendoza (ed.); $2^{\circ}$ ).

BICU. (2011). Estudio de Caso: Proyecto Validación de Buenas Prácticas Agrícolas en el Cultivo de Palma Africana en Kukra Hill , RAAS , Nicaragua. 48.

Cadenas, I. (2008). Fitopatología General. Dpto. academico de Entomologia y Ftoptologia, 1, 78. 


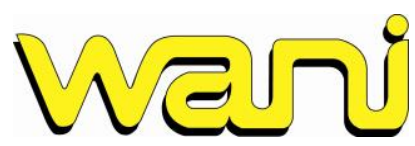

Revista del Caribe Nicaragüense

Núm. 76 | enero - junio, 2022
e-ISSN 2308-7862

Bluefields Indian \& Caribbean University (BICU)

DOI: https://doi.org/10.5377/wani.v38i76.13377 http://revistas.bicu.edu.ni/index.php/wani wani@bicu.edu.ni

Cave, R. D., Trabanino, R., \& Pitty, A. (2013). Zamorano y sus Contribuciones a la Agricultura Sostenible a Través del Control Biológico de Plagas. Ceiba. https://doi.org/10.5377/ceiba.v52i1.966

Chinchilla, C. M. (2003). Manejo integrado de problemas fitosanitarios en Elaeis guineensis en América Cetnral. Experiencias, 67, 69-82.

Diez Casero, J. J. (2013). Patologías Forestales y Cambio Global: Globalización, Cambio Climático y Cuestiones legales. $\sigma^{\circ}$ Congreso Forestal Español, 258, 13. https://doi.org/10.1017/CBO9781107415324.004

Echandi, E. (1967). Manual De Laboratorio Para Fitopatologia General. 54.

Flores-Pacheco, J.A., Mairena, A., \& Espluga, J. (2013). Evaluación de riesgos en sistemas agrícolas asociados a la utilización de plaguicidas en el Municipio de Kukra Hill, Nicaragua, Centroamérica. Nexos Revista científica, 26(ISSN-L 1818-6742), 34-44. https://doi.org/http://dx.doi.org/10.5377/nexo.v26i1.1034

Flores-Pacheco, Juan Asdrubal, Aguirre Median, S. M., Taylor Britton, D. M., \& Cassell's Martínez, R. A. (2018). Evaluación de trampas con atrayentes para la captura de dípteros. Revista Científica de FAREM-Estelí, 27, 38-51. https://doi.org/10.5377/farem.v0i27.7057

Franco, F. (2004). Muestreo de nematodos en banano.

Landero-Torres, I., Presa-Parra, E., Galindo-Tovar, M. E., Leyva-Ovalle, O. R., MurguíaGonzález, J., Valenzuela-González, J. E., \& García-Martínez, M. Á. (2015). Variación Temporal y Espacial de la Abundancia del Picudo Negro ( Rynchophorus palmarum L., Coleoptera: Curculionidae) en Cultivos de Palmas Ornamentales del Centro de Veracruz, México . Southwestern Entomologist. https://doi.org/10.3958/059.040.0116

Lastres, L., \& Soza, F. (2009). Manual Sanidad Vegetal. Programa de Manejo Integrado de Plagas en América Central "PROMIPAC", 75.

Leslie, J. F., Summerell, B. A., \& Bullock, S. (2006). The Fusarium Laboratory Manual, Online Book Version. The Fusarium Laboratory Manual, 388. http://sfx.hbznrw.de/sfx_ubo?ctx_ver=Z39.88-2004\&ctx_enc=info:ofi/enc:UTF-

8\&rfr_id=info:sid/summon.serialssolutions.com\&rft_val_fmt=info:ofi/fmt:kev:mtx:book\&r ft.genre=book\&rft.title=The fusarium laboratory manual\&rft.au=Bullock, Suzanne\&rft.au=Leslie, $\mathrm{J}$

López, R. (1980). Determinación de los nematodos fitoparasitos asociados al plátano en Río Frío. En Agronomia Costarricense (Vol. 4, Número 2, pp. 143-157).

Luc, M., Sikora, R. A., \& Bridge, J. (2005). Plant parasitic nematodes in subtropical and tropical agriculture. En Institute of Parasitology. https://doi.org/10.1079/9780851997278.0000

Mendonça, R. S., Navia, D., \& Flechtmann, C. H. W. (2007). Raoiella indica. Embrapa Recursos Genéticos e Biotecnologia.

Oehlschlager, A. C., Chinchilla, C., Castillo, G., \& Gonzalez, L. (2006). Control of red ring disease by mass trapping of Rhynchophorus palmarum (coleoptera: curculionidae). Florida Entomologist. https://doi.org/10.1653/0015-4040(2002)085[0507:corrdb]2.0.co;2

Perry, R. N., \& Moens, M. (2013). Plant Nematology. En Soil Science (Vol. 106, Número 4). https://doi.org/10.1097/00010694-196810000-00022

REPcar - BICU. (2014). Proyecto GEF-REPCar : reduciendo el escurrimiento Buenas Prácticas 
Agrícolas y Monitoreo del Escurrimiento ii; Por un Ambiente Saludable ... ... Produzcamos sin contaminar!!!

Serra, C. A., García, S., Ferreira, M. A., Batista, O., Epsky, N. D., \& Heath, R. R. (2005). Comparación de atrayentes para el trampeo de moscas de las frutas, Anastrepha spp. (Diptera: Tephritidae) en frutales en la republica dominicana. Proceedings of the Caribbean Food Crops Society., XXXXI(2), 524-532.

Sommer, R. J., Carta, L. K., \& Sternberg, P. W. (1994). The evolution of cell lineage in nematodes. Development (Cambridge).

Taussig, M. (2018). Palma Africana. En Palma Africana. https://doi.org/10.7208/chicago/9780226516271.001.0001 